\title{
AIE-Active Poly(phenyleneethynylene)s for Fluorescence and Raman Dual-Modal Imaging and Drug-Resistant Bacteria Killing
}

Xiang Su, Ruihua Liu, Ying Li, Ting Han*, Zhijun Zhang, Niu Niu, Miaomiao Kang, Shuang Fu, Dong Wang*, and Ben Zhong Tang*

Dr. X. Su, Dr. Y. Li, Dr. T. Han, Dr. Z. Zhang, Dr. N. Niu, Dr. M. Kang, Dr. S. Fu, Prof. D. Wang

Center for AIE Research, Shenzhen Key Laboratory of Polymer Science and Technology, Guangdong Research Center for Interfacial Engineering of Functional Materials, College of Materials Science and Engineering, Shenzhen University, Shenzhen 518060, China

E-mail: hanting@szu.edu.cn,wangd@szu.edu.cn

Dr. X. Su, Dr. Z. Zhang, Dr. N. Niu, Dr. M. Kang, Dr. S. Fu

College of Physics and Optoelectronic Engineering, Shenzhen University, Shenzhen 518060, China

Dr. X. Su, Prof. B. Z. Tang

Department of Chemistry, Hong Kong Branch of Chinese National Engineering

Research Center for Tissue Restoration and Reconstruction, The Hong Kong

University of Science and Technology, Clear Water Bay, Kowloon, Hong Kong, China

Prof. R. Liu

State Key Laboratory of Medicinal Chemical Biology and College of Life Sciences, Nankai University, Tianjin 300071, China

Prof. B. Z. Tang

Shenzhen Institute of Aggregate Science and Technology, School of Science and Engineering, The Chinese University of Hong Kong, Shenzhen, Guangdong 518172, China

E-mail: tangbenz@cuhk.edu.cn

Keywords: aggregation-induced emission, poly(phenyleneethynylene)s, fluorescence-Raman imaging, luminescent antibacterial agent 


\section{Abstract}

Poly(phenyleneethynylene) (PPE) is a widely used functional conjugated polymer with applications ranging from organic optoelectronics and fluorescence sensors to optical imaging and theranostics. However, the fluorescence efficiency of PPE in aggregate states is generally not as good as their solution states, which greatly compromises their performance in fluorescence-related applications. Herein, we design and synthesize a series of PPE derivatives with typical aggregation-induced emission (AIE) properties. In these PPEs, the diethylamino-substituted tetraphenylethene units function as the long-wavelength AIE source and the alkyl side chains serve as the functionalization site. The obtained AIE-active PPEs with large $\pi$-conjugation show strong aggregate-state fluorescence, interesting self-assembly behaviors, inherently enhanced alkyne vibrations in the Raman-silent region of cells, and efficient antibacterial activities. The PPE nanoparticles with good cellular uptake capability can clearly and sensitively visualize the tumor region and residual tumors via their fluorescence and Raman signals, respectively, to benefit the precise tumor surgery. After post-functionalization, the obtained PPE-based polyelectrolyte can preferentially image bacteria over mammalian cells and possesses efficient photodynamic killing capability against Gram-positive and drug-resistant bacteria. This work provides a feasible design strategy for developing multifunctional conjugated polymers with multimodal imaging capability as well as photodynamic antimicrobial ability. 


\section{Introduction}

Conjugated polymers (CPs) is an important category of functional materials and have attracted worldwide interest for many years. The presence of alternant $\sigma$ and $\pi$-bonds in CP backbones remarkably promotes the $\pi$-electron delocalization along the conjugated chains and endows CPs with unique photophysical, electrical, mechanical, and chemical properties. ${ }^{[1-5]}$ In the past decades, CPs have been applied in diverse areas, ranging from light-emitting diodes, ${ }^{[6]}$ field-effect transistors,${ }^{[7]}$ organic solar cells ${ }^{[8]}$ flexible energy harvesting devices ${ }^{[9]}$ to fluorescence sensors ${ }^{[10]}$ and biomedical applications. ${ }^{[11-12]}$ As one of the most widely used CPs, poly(phenyleneethynylene)s (PPEs) are chemically stable and can be readily synthesized by Sonogashira coupling reactions. The unique linear and rigid conjugated backbones make PPEs excellent materials in chemo-/bio-sensing, ${ }^{[13-14]}$ optical imaging and phototherapy, ${ }^{[15-17]}$ etc. For example, PPE-based fluorescence sensors can afford "million-fold" signal amplification toward analytes due to the molecular wire effect. PPE-based conjugated polyelectrolytes have been applied as fluorescence imaging-guided antibacterial agents. ${ }^{[18-19]}$ However, the fluorescence efficiencies of PPE aggregates are generally not as good as their solution states, which greatly compromises their performance in fluorescence-related applications. The weak aggregate-state fluorescence could be ascribed to the easy formation of detrimental species such as excimers because the rigid rod-like backbones of PPEs tend to experience strong $\pi$ - $\pi$ stacking interactions upon aggregation. ${ }^{[20]}$ Although great efforts have been devoted to improving the fluorescence efficiency of PPEs in aggregate states, ${ }^{[21-22]}$ the existing methods 
generally requires the difficult and tedious synthesis of bulky monomers or needs to passively avoid the formation of aggregates. Therefore, the development of facile and efficient strategy toward multifunctional PPEs with excellent aggregate-state fluorescence is highly desirable.

Materials with aggregation-induced emission (AIE) properties possess highly efficient fluorescence in aggregate states, ${ }^{[23-24]}$ which may provide a good solution to the above-mentioned fluorescence quenching problem of PPEs. AIE luminogens (AIEgens) generally possess propeller-shaped conformations with freely rotatable aromatic rings or vibratile structures. The twisted conformation effectively hampers the intermolecular $\pi-\pi$ stacking interactions and the active intramolecular motions of multiple rotors or vibrators are restricted upon aggregation. These two effects collectively promote the radiative decay of excited states and thus result in the enhanced fluorescence efficiency in aggregate states. Till now, a variety of AIEgens with easily modified structures and facile synthetic routes have been developed, including tetraphenylethene (TPE), distryreneathracence, tetraphenylpyrazine, etc. Therefore, we anticipated that conjugated PPEs with excellent aggregate-state fluorescence as well as multiple functionalities might be developed by introducing such AIEgens into the PPE main chains.

The combination of AIEgen with PPE could be a win-win design. With the inherent advantages of PPEs and AIEgens, AIE-active PPEs might show unique performance and functionalities that are hard to be achieved by conventional CPs and AIE materials. For example, AIEgens are widely used in fluorescence bio-imaging 
with the advantages of high photostability, high fluorescence efficiency, and excellent biocompatibility. Fluorescence imaging (FLI) technique generally possesses the merits of simplicity, high sensitivity, non-invasiveness, rapid response, and real-time monitoring. However, the fluorescence signal of AIEgens is often susceptible to biological microenvironments and is easily interfered by the autofluorescence background of cells and tissues, thus leading to the limited precision and spatial resolution. ${ }^{[25-26]}$ On the other hand, Raman imaging (RI) features narrow bands with stable Raman intensities at a cell-silent region $\left(1800-2800 \mathrm{~cm}^{-1}\right)$, consequently achieving the high-resolution imaging without the interference of biological background. ${ }^{[27]}$ Nevertheless, RI often suffers from low sensitivity due to the weak Raman signals. To magnify the Raman signals of Raman probes, advanced Raman-enhancing techniques such as coherent anti-Stokes Raman scattering, ${ }^{28-29]}$ surface-enhanced Raman scattering, ${ }^{[30-31]}$ and stimulated Raman scattering ${ }^{[32-33]}$ are often required. Another more practical and facile strategy is developing probes with inherently enhanced Raman signals by rational molecular design. ${ }^{[34-36]}$ An effective design principle for Raman enhancement is extending the $\pi$-conjugation units to strengthen the stretching and vibration characteristics of triple bonds. Following this principle, conjugated PPE derivatives has been demonstrated to possess inherently enhanced alkyne vibrations in the Raman-silent region of cells compared to alkyne-containing small molecules. ${ }^{[37]}$ Therefore, PPE derivatives are potential to serve as good Raman-responsive materials for enhanced Raman imaging of living biological system. Taking the complementary advantages of AIEgen and PPE in FLI 
and RI techniques, we proposed that AIE-active conjugated PPEs are likely to be a new type of FLI-RI dual-modal imaging agent to benefit comprehensive disease diagnosis and treatment. Although a few AIE-based FLI-RI agents have been reported, ${ }^{[38-39]}$ these cases were all focused on small molecules. The examples of one-for-all polymer-based imaging agents with both efficient aggregate-state fluorescence and enhanced Raman properties in cell-silent region still remain very rare. Therefore, the study on the application of AIE-active PPEs on fluorescence and Raman dual-modal imaging is necessary and may open a new way for developing efficient polymer-based multi-modality imaging agents.

Moreover, by introducing hydrophilic charged side chains to the hydrophobic $\pi$-conjugated skeletons, the derived AIE-active PPEs could be endowed with other interesting properties and functionalities, such as diverse assembly morphologies and efficient antimicrobial activities. Bacterial infections have brought great threats to human beings' health. To defeat pathogenic bacteria, antibiotics have been widely used due to their excellent bacteria-killing capabilities. However, the extensive use of antibiotics gave rise to the rapid emergency and diffusion of multidrug-resistant bacteria, which has become a serious global healthcare problem. Compared to developing new antibiotics, a more desirable strategy is exploring alternative antibacterial agents that are hard to develop resistance. To meet this requirement, scientists have developed bacteriophages, ${ }^{[40-41]}$ quaternary ammonium compounds, ${ }^{[42]}$ and antimicrobial peptides, ${ }^{[43]}$ etc. In recent years, photodynamic therapy (PDT) reagents including both AIEgens and water-soluble CPs have been applied in 
drug-resistant bacteria killing with the features of minimal invasiveness and side effects, low cytotoxicity and free of drug resistance. ${ }^{[44-51]}$ For instance, cationic PPEs with quaternary ammonium pendant can preferentially interact with bacterial outer cell membranes and effectively killbacteria by generating toxic reactive oxygen species (ROS) upon irradiation. ${ }^{[18-19,46,51-52]}$ AIEgen-based photosensitizers not only are good antibacterial agents, $\left[{ }^{44-45}\right]$ but can also achieve ultrafast bacterial imaging with low background and good sensitivity without extra washing steps. Hence, the introduction of AIE characteristics to cationic PPE-based photosensitizers could be a promising design strategy for developing multifunctional CPs with excellent imaging capability as well as efficient biocidal capability against a variety of bacteria.

For the above reasons, herein we designed and synthesized a series of AIE-active PPE derivatives named CP0-CP2, in which the diethylamino-substituted TPE unit serve as the long-wavelength AIEgen source and the alkyl side chains serve as the functionalization site (Figure 1). Due to the combination of PPE with AIEgen, the obtained CPs were demonstrated to possess multiple excellent properties including intrinsic Raman enhancement of alkyne units, strong aggregate-state fluorescence, unique self-assembly behaviors, and efficient antibacterial activities. Attracted by these properties, the applications of CP0-CP2 in FLI-RI dual-modal imaging-guided tumor surgery, bacteria imaging, and PDT-promoted killing of drug-resistant bacteria were carefully investigated in this work. 


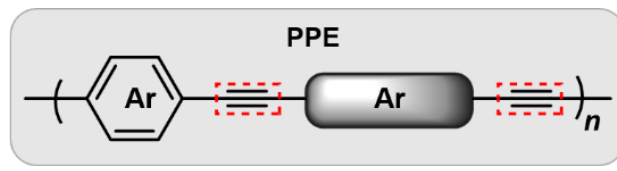

Intrinsic Raman enhancement
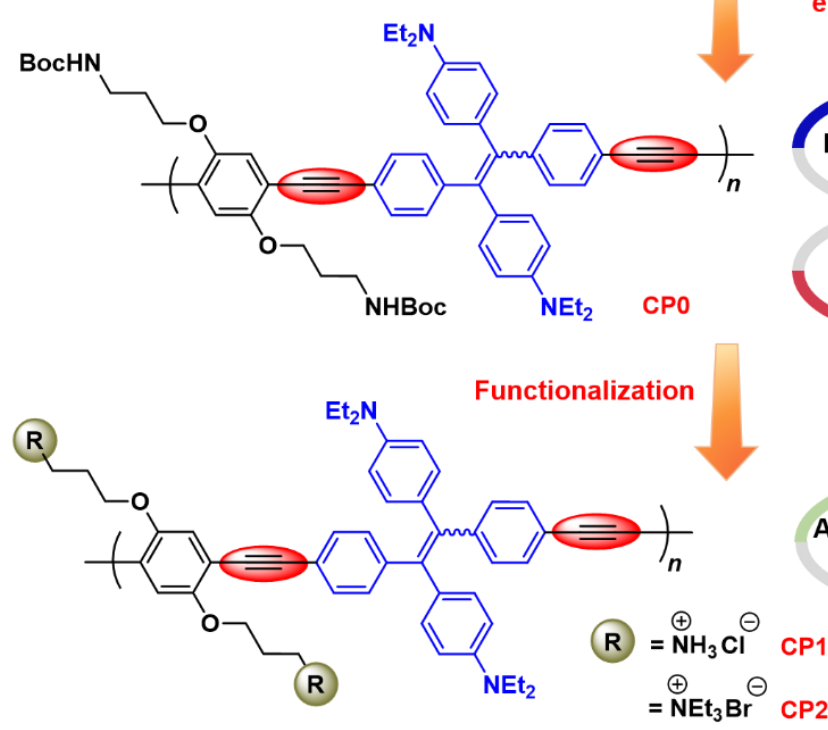

Fluorescence enhancement

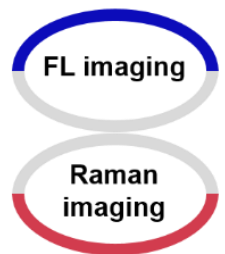

Antibacterial activity

Figure 1. Schematic illustration of the design strategy for multifunctional AIE-active PPEs.

\section{Results and Discussion}

\subsection{Synthesis and Characterization}

TPE is a typical AIEgen with a high fluorescence efficiency in aggregate states but its short absorption and emission wavelengths are unfavorable for bioimaging applications. To fulfill our research purpose, $N, N$-diethylamino-substituted TPE unit with relatively longer absorption and emission wavelengths ${ }^{[53]}$ was selected and introduced into the PPE backbones. First, the diethylamino-substituted TPE diyne was prepared according to the synthetic routes shown in Scheme S1. Then polymer CP0 with tert-butyloxycarbonyl (Boc) groups and polymer CP2 with quaternary ammonium pendants were readily prepared through palladium-catalyzed Sonogashira coupling reactions of the AIEgen-containing diyne and different alkyl 
chain-containing aryl diiodides (Scheme S2). Polymer CP1 with positive ammonium groups was synthesized by the facile post-modification reaction of $\mathbf{C P 0}$ to deprotect the Boc group under acidic conditions. The structures of monomers and CP0-2 were all confirmed by NMR characterization (Figure S1-S3). Taking CP0 as an example, the ${ }^{1} \mathrm{H}$ NMR spectrum of $\mathbf{C P 0}$ showed no resonance signals matching with the resonance peaks of the acetylene protons of the diyne monomer at $\delta 3.07-3.03$. Instead, the characteristic peaks associated with the diethylamino-substituted TPE unit and the alkyl chains were observed in the spectrum of $\mathbf{C P 0}$ (Figure S1). These results suggested that the monomers were completely converted into polymers with desired structures. Polymer CP0 possesses good solubility in commonly used organic solvents, such as DCM, THF, $\mathrm{CHCl}_{3}$, acetone, etc., whereas $\mathbf{C P 1}$ and $\mathbf{C P 2}$ are only soluble in strong polar organic solvents like $\mathrm{MeOH}$, DMSO and DMF. The water solubility of CP1 is much better than that of CP2.

\subsection{Aggregation-Induced Emission}

The photoluminescence (PL) behaviors of CP0-2 in different states were carefully investigated. As shown in Figure S4, the absorption spectra of CP0-2 peaked at a similar wavelength of about $430 \mathrm{~nm}$. The potential AIE properties of these three PPE derivatives were studied in different solvent mixtures depending on their respective solubility. As depicted in Figure 2A and 2D, CPO showed typical AIE features in $\mathrm{THF} / \mathrm{H}_{2} \mathrm{O}$ mixtures. With the gradual addition of water, the PL intensity of CPO kept rising and reached the maximum at a water fraction of $80 \%$. Further increasing the water content to $90 \%$ led to a slight decrease in PL intensity, which might result from 
the reduced effective solute concentration due to the extensive formation of aggregates. The aggregates and solid powders of $\mathbf{C P 0}$ showed strong red fluorescence at about $630 \mathrm{~nm}$. And the fluorescence quantum yield of $\mathbf{C P 0}$ in pure THF solution and $\mathrm{THF} /$ water mixture with $90 \%$ water fraction was measured to be $1.05 \%$ and $11.37 \%$, respectively, which further confirmed its AIE property. The water soluble polymer CP1 behaved differently from CP0 regarding the PL property in aggregate states. As shown in Figure 2B, the water solution of CP1 was faintly emissive with a maximum emission wavelength at about $640 \mathrm{~nm}$. Along with the addition of THF fraction, the PL intensity of CP1 was rapidly enhanced with a 14-fold increase at $40 \%$ THF fraction. When the THF fraction was further increased to above $70 \%$, a slight drop in the PL intensity of CP1 was observed. Interestingly, although the nanoaggregates of CP1 in water/THF mixtures showed red fluorescence, the loose macroaggregates of $\mathbf{C P 1}$ that are freshly precipitated in acetone during the preparation process showed very strong yellow-green fluorescence. After vacuum drying, the obtained dense solid powders were almost non-emissive except for some tiny spots around the edges. These results indicated that the surrounding solvent environments and packing density of aggregates may insert a great influence on the PL behaviors of CP1. Similar conclusion can also be drawn from the PL results of CP1 in DMSO/toluene mixtures (Figure S5). The significantly decreased fluorescence of CP1 in solid state might be due to the low steric hindrance of ammonium salt groups, which may favor the formation of strong inter- and intra-chain $\pi-\pi$ interactions in densely packing modes and thus quench the fluorescence. By replacing 
ammonium groups with bulky quaternary ammonium pendants, the aggregate and solid-state fluorescence was remarkably enhanced both in stability and in efficiency. As depicted in Figure 2C, the aggregates and solid powders of CP2 showed intense red fluorescence. The weak fluorescence of its dilute DMSO solution was gradually enhanced by adding the poor solvent (toluene) to induce the formation of aggregates, demonstrating an obvious AIE characteristic.
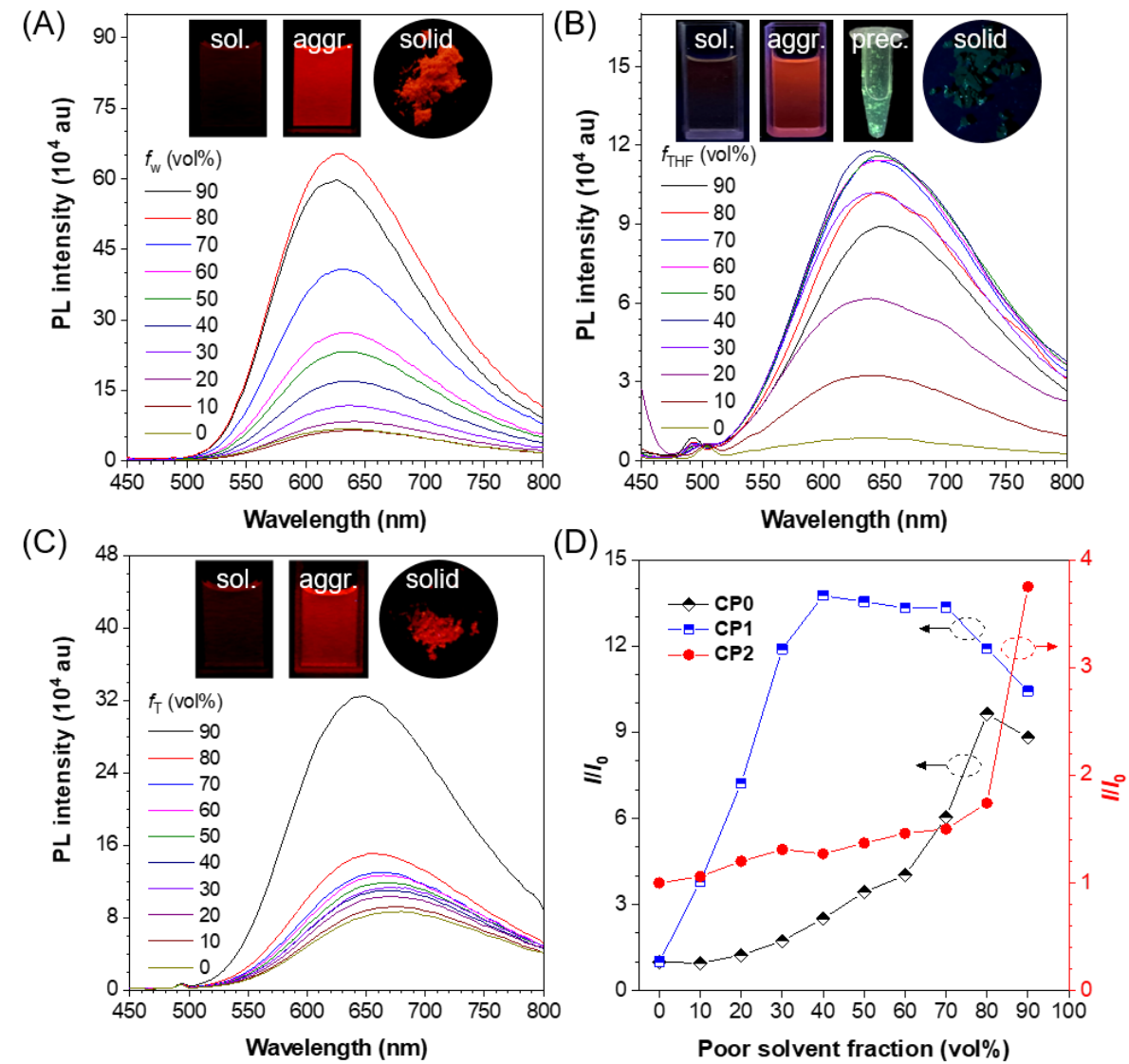

Figure 2. (A-C) Emission spectra of (A) $\mathbf{C P 0}$ in $\mathrm{THF} / \mathrm{H}_{2} \mathrm{O}$, (B) CP1 in $\mathrm{H}_{2} \mathrm{O} / \mathrm{THF}$, and (C) CP2 in DMSO/toluene mixtures with different water, THF or toluene fractions $\left(f_{\mathrm{w}}, f_{\mathrm{THF}}\right.$ or $\left.f_{\mathrm{T}}\right)$, respectively. (D) Plots of the relative PL intensity $\left(I / I_{0}\right)$ versus the composition of the solution mixtures of CP0-2. Inset: fluorescent photographs of (A) CPO in THF solution, THF/water mixture with $90 \%$ water fraction, and solid state, (B) CP1 in water solution, water/THF mixture with 90\% THF fraction, precipitate in acetone over the preparation of CP1, and solid state, (C) CP2 in DMSO solution, DMSO/toluene mixture with $90 \%$ toluene fraction, and solid state. The photographs were taken under $365 \mathrm{~nm}$ UV irradiation. $I_{0}=$ intensity at pure solutions. Solution concentration: $10 \mu \mathrm{g} / \mathrm{mL}$. Excitation wavelength $=435 \mathrm{~nm}$ (for CP0) and $430 \mathrm{~nm}$ (for CP1 and CP2). 
The interesting PL properties of CP1 in aggregate states attracted us to gain insight into its aggregation behaviors at different solvent environments to assist the mechanism explanation. The self-assembly morphologies of CP1 with a concentration of $10 \mu \mathrm{g} / \mathrm{mL}$ in water/THF mixtures were carefully characterized by SEM, TEM, AFM, and stimulated emission of depletion (STED) nanoscopy (Figure 3 and Figure S6). The SEM image shown in Figure 3A suggested that inhomogeneous particles were formed in water/THF mixtures with a THF fraction $\left(f_{\mathrm{THF}}\right)$ of $20 \%$. Further increasing $f_{\mathrm{THF}}$ to $40 \%$ led to much more regular spherical particles with diameters ranged from $\sim 20$ to $\sim 100 \mathrm{~nm}$ (Figure 3B). The easy formation of aggregates upon the addition of poor solvent might origin from the presence of multiple inter- or intra-chain interactions between the hydrophobic TPE-containing rigid backbone and the hydrophilic flexible side chains. At a $f_{\mathrm{THF}}$ of $60 \%$, these spherical particles may be inclined to get closer and coalesce one after another through their efficient intra- or inter-chain interactions, thus resulting in "necklace"-like morphologies sprinkled with some nanofibers (Figure 3C). ${ }^{[54-55]}$ When the $f_{\mathrm{THF}}$ reached $80 \%$, the previous “necklace"-like morphologies gradually turned into many twisted helical nanofibers as observed in Figure 3D. The locally enlarged image shown in Figure 3E clearly demonstrated the helical structure of the fibers with a width of $\sim 50 \mathrm{~nm}$. Additionally, some winding coil structures were also observed in this sample probably due to the bending and entanglement of helical fibers (Figure 3F). The TEM and AFM analysis also revealed the apparent helix structure of the self-assembly nanofibers with a helical pitch of $\sim 100 \mathrm{~nm}$ (Figure 3G-H and Figure S6). More importantly, thanks to 
the fluorescence property of CP1, these helical fibers can also be visualized by STED nanoscopy. As depicted in Figure 3I, fluorescent nanofibers with helical morphology were observed with a good signal-to-noise ratio. Similar self-assembly behaviors were also observed at a low concentration of $1 \mu \mathrm{g} / \mathrm{mL}$ CP1 (Figure S7). The diversiform self-assembly morphologies of the amphiphilic CP1 could be the collective result of multiple interactions, including hydrophobic effect, electrostatic interaction, and noncovalent interactions such as $\pi-\pi$ interactions and hydrogen bonding. ${ }^{[54-57]}$ Compared with the relatively loose accumulation of inhomogeneous particles at a $f_{\mathrm{THF}}$ of 20\%, CP1 in spherical particles and helical fibers may take a much tighter stacking mode to further restrict the intramolecular motions of the AIEgen unit, thus leading to a further enhancement in the aggregate-state fluorescence efficiency. This morphological transformation was consistent with the PL change tendency of CP1 shown in Figure 2B. When the packing of CP1 chains became very dense such as in dried solid state, the formation of strong $\pi-\pi$ interactions between polymer chains might play a more important role than the AIE effect in the PL property of CP1 and consequently caused the fluorescence quenching. 

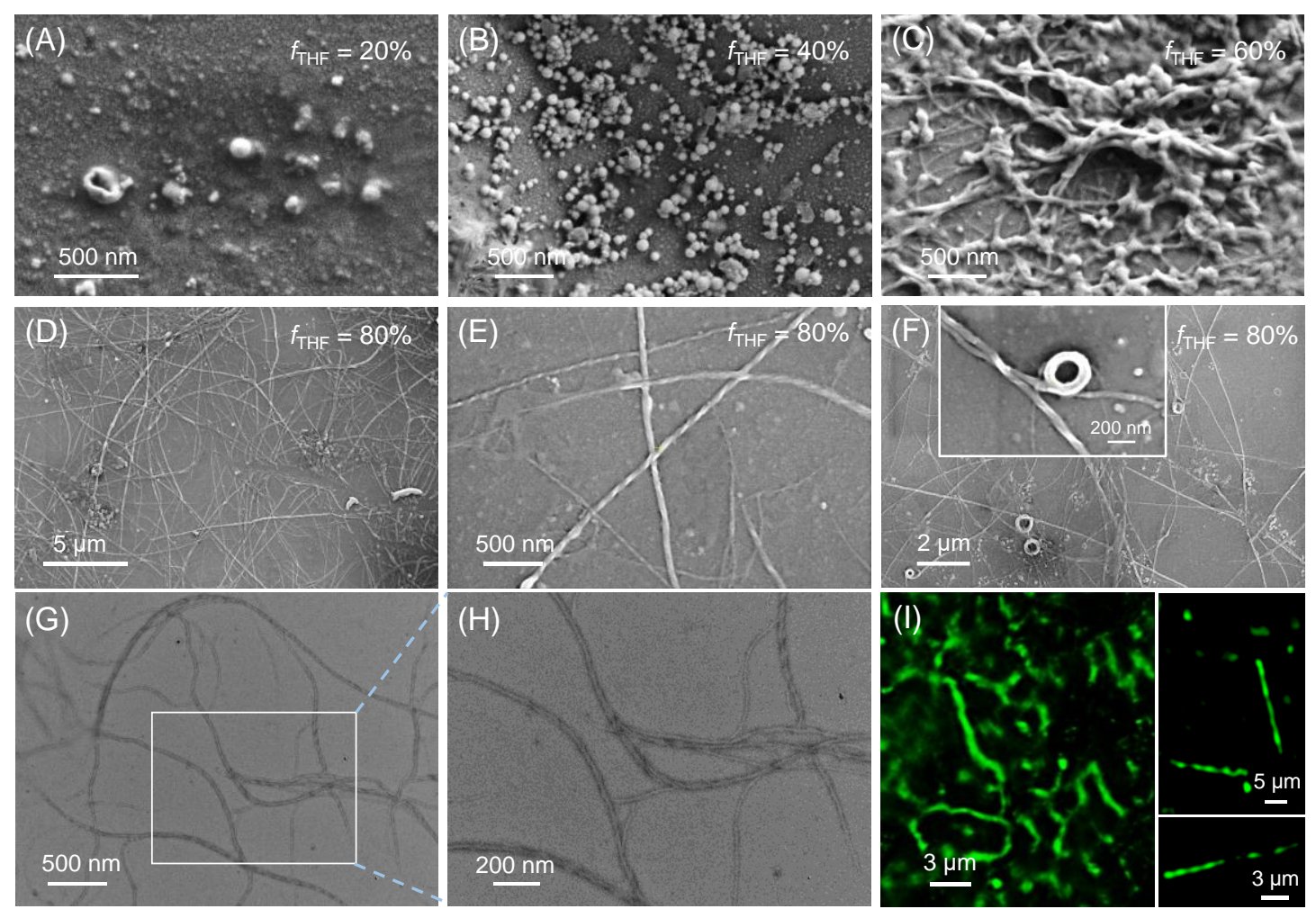

Figure 3. Diversiform self-assembly morphologies of CP1. SEM images of CP1 (10 $\mu \mathrm{g} / \mathrm{mL}$ ) in $\mathrm{H}_{2} \mathrm{O} / \mathrm{THF}$ mixtures with a THF fraction $\left(f_{\mathrm{THF}}\right)$ of (A) $20 \%$, (B) $40 \%$, (C) $60 \%$ and $(\mathrm{D}-\mathrm{F})$ 80\%. The inset image in $(\mathrm{F})$ is the high-resolution image of the winding coil structure. ( $\mathrm{G}$ and $\mathrm{H}$ ) TEM images of helical nanofibers. (I) Fluorescence images of helical nanofibers captured by STED nanoscopy.

\subsection{Enhanced Raman properties}

Alkyne group has a distinct Raman scattering peak in the cellular silent region (1800-2800 $\mathrm{cm}^{-1}$ ) but the inherent alkyne vibrations of alkyne-containing small molecules are often not sufficient enough for high-contrast Raman imaging. The rigid backbone and delocalized $\pi$-conjugated structure of PPE derivatives are advantageous to enhance the Raman intensity of alkyne groups. ${ }^{[37]}$ Investigation of the relative intensities in contrast with a standard Raman tag under the same conditions is a well-accepted method to compare Raman intensities among different Raman-active probes. As a stable and nonvolatile live-cell Raman imaging reagent, 5-ethynyl-2'-deoxyuridine (EdU) is widely utilized as a standard alkyne Raman tag. 
The Raman-enhanced capability of the designed polymers was then evaluated using the relative Raman intensity versus EdU (RIE) as evaluating indicator. Taking CP0 as a representative example, the RIE value of $\mathbf{C P 0}$ and the diethylamino-substituted TPE diyne monomer (M1) were calculated from the peak area of respective alkyne peaks (Figure 4). As presented in Figure 4B, the alkyne peak of CP0 and M1 located at $\sim 2210 \mathrm{~cm}^{-1}$ and $\sim 2110 \mathrm{~cm}^{-1}$, respectively, and the Raman intensity of CP0 was obviously higher than the corresponding small molecules under the same conditions. The RIE value CP0 was calculated to be 17.1, whereas the RIE of M1 was merely 3.6, showing a polymerization-induced Raman enhancement effect. Meanwhile, we also compared the Raman property of $\mathbf{C P 0}$ with other traditional strong Raman tags such as conjugated alkyne (diphenylacetylene, DPA) and diacetylene derivative (buta-1,3-diyn-1-ylbenzene, BDB). The results shown in Figure 4C suggested that the alkyne Raman scattering of CP0 was much stronger than the previously reported small molecule Raman tags. Combined with the abovementioned efficient aggregate-state fluorescence, it is thus worthy investigating the application of the obtained AIE-active CPs in FLI-RI dual-modal imaging-guided tumor surgery. 

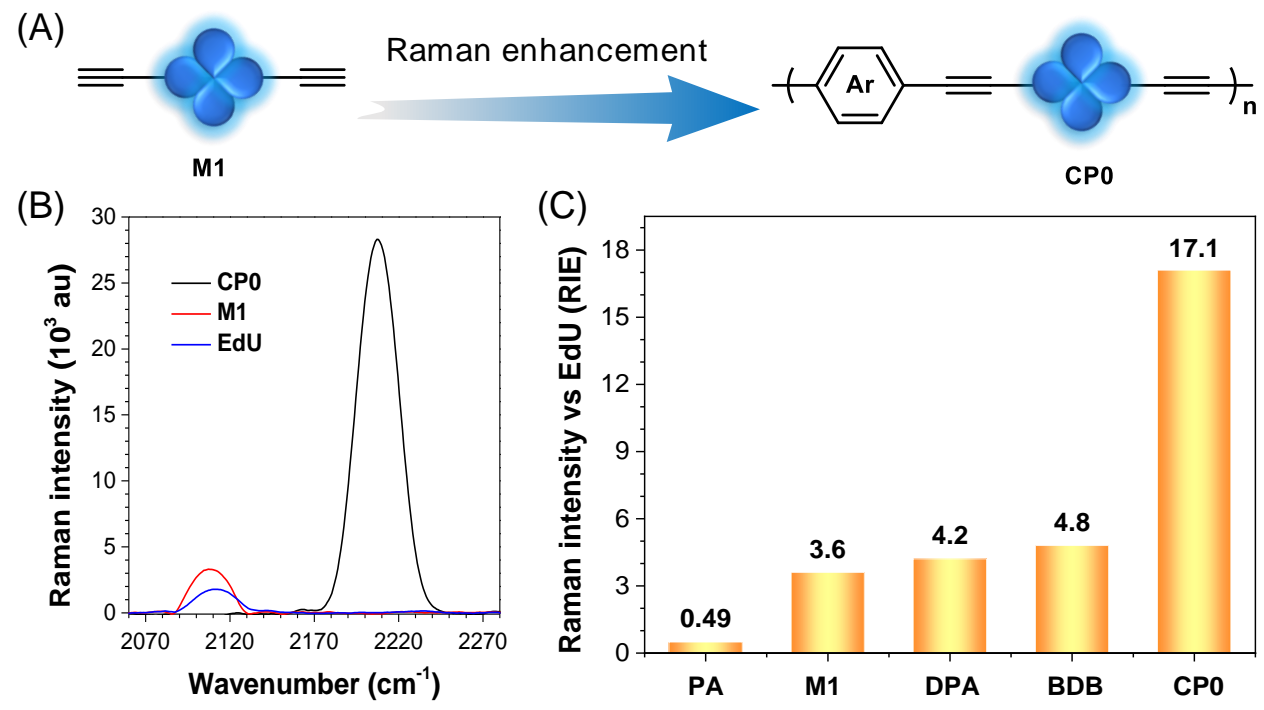

Figure 4. (A) Schematic illustration of the polymerization-induced Raman enhancement from M1 to CP0. (B) Overlaid Raman spectra of individual solution of CP0 and M1 in THF and EdU in DMSO (10 mM, excitation wavelength: $785 \mathrm{~nm})$. (C) Relative Raman intensity versus EdU (RIE) of CP0, M1 and other reported representative alkynes $(\mathrm{PA}=$ phenylacetylene; $\mathrm{DPA}=$ diphenylacetylene; $\mathrm{BDB}=$ buta-1,3-diyn-1-ylbenzene).

\subsection{Fluorescence and Raman dual-modal imaging}

CP0 and CP2 were selected for the subsequent biological applications considering their more steady and efficient aggregate-state emission than CP1. As a preliminary study, in vitro cell imaging experiments were conducted using $\mathbf{C P 0}$ and $\mathbf{C P 2}$ to estimate their cellular uptake capability and imaging brightness. To improve the dispersibility and compatibility of $\mathbf{C P 0}$ in cellular and tumor environments, the hydrophobic CP0 was encapsulated with amphiphilic DSPE- $m$ PEG2000 to form stable and water-soluble nanoparticles (NPs) through a conventional nanoprecipitation process. The average size of CP0 NPs was measured by dynamic light scattering with a mean diameter size of $109.8 \mathrm{~nm}$ and a small PDI value 0.072 (Figure S8A). Similar to $\mathbf{C P 0}$ solution, CP0 NPs also exhibited a conspicuous and narrow Raman band at 
around $2210 \mathrm{~cm}^{-1}$, indicating the good stability of Raman properties (Figure S8B). As shown in Figure S9, CP0 NPs can efficiently enter HeLa cells to show bright red fluorescence under the excitation of $405 \mathrm{~nm}$ light. By contrast, the cell-uptake efficiency of $\mathbf{C P 2}$ was not satisfactory even after prolonging the incubation time to 4 h (Figure S10). The poor cellular uptake capability of CP2 might be due to the relatively strong electrostatic interactions between the cationic CP2 and the negative charged cell membrane. ${ }^{[18]}$

We then employed CP0 NPs for the following in vivo tumor imaging experiments. Initially, fluorescence imaging (FLI) was performed by tail intravenous injection of CP0 NPs into tumor-bearing mice. As depicted in Figure S11A-B, the fluorescence intensity in the tumor site gradually increased during $0-9 \mathrm{~h}$ of the post-injection and then decreased over time due to the metabolism. Although CP0 NPs could image the tumor site via fluorescence, the strongest signal was observed in the liver region no matter for in vivo imaging or in separated organs (Figure S11C-D). To gain high-contrast and clearly distinguishable tumor imaging, we thus conducted time-dependent FLI by intratumor injection of CP0 NPs. As depicted in Figure 5A, a clear and bright visualization of the tumor region can be achieved after intratumor injection of CP0 NPs. Obvious fluorescence signal can still be detected from the tumor site even after injection for $24 \mathrm{~h}$. Taking advantage of the good FLI performance and enhanced Raman properties of CP0, we next investigated its application in the fluorescence and Raman dual-modal imaging-guided tumor resection surgery. The first surgery (S1) on a tumor-bearing mouse was conducted 
after injection of CP0 NPs for $24 \mathrm{~h}$ based on the preoperative fast and non-invasive FLI technique. After the S1 tumor excision (post S1), however, a certain degree of fluorescence signal was detected in the FLI result of post S1, indicating that there might be residual tumors left behind (Figure 5B). Owing to the relatively low signal-to-background ratio and environmental susceptibility of FLI, it is difficult to distinguish the fluorescence stemming from the NPs in residual tiny tumors or the autofluorescence of normal tissues. Based on the high Raman signal of CP0 NPs in cell-silent region, Raman imaging (RI) with microscopic resolution was then performed in the suspicious region to assist precise surgery. As presented in Figure 5C, the RI result of alkyne vibrations of CP0 NPs can sensitively visualize the residual tumors and their boundaries to normal tissues in the faint fluorescent region, which was also confirmed via further H\&E histological analysis (Figure 5D). After precisely positioning the residual tumors, a second surgery (S2) was then performed to remove them until no fluorescence and Raman signals could be detected (Figure 5E-G). These results verified our design principle by demonstrating that the AIE-active conjugated PPEs are promising FLI-RI dual-modal imaging agents to benefit the precise cancer surgery. 
(A)
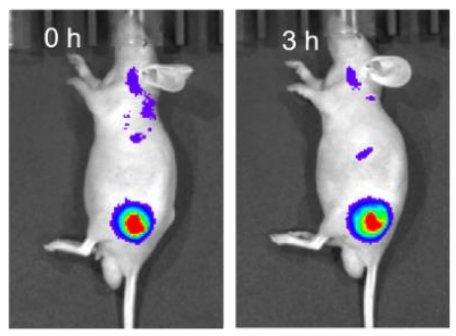

(B)
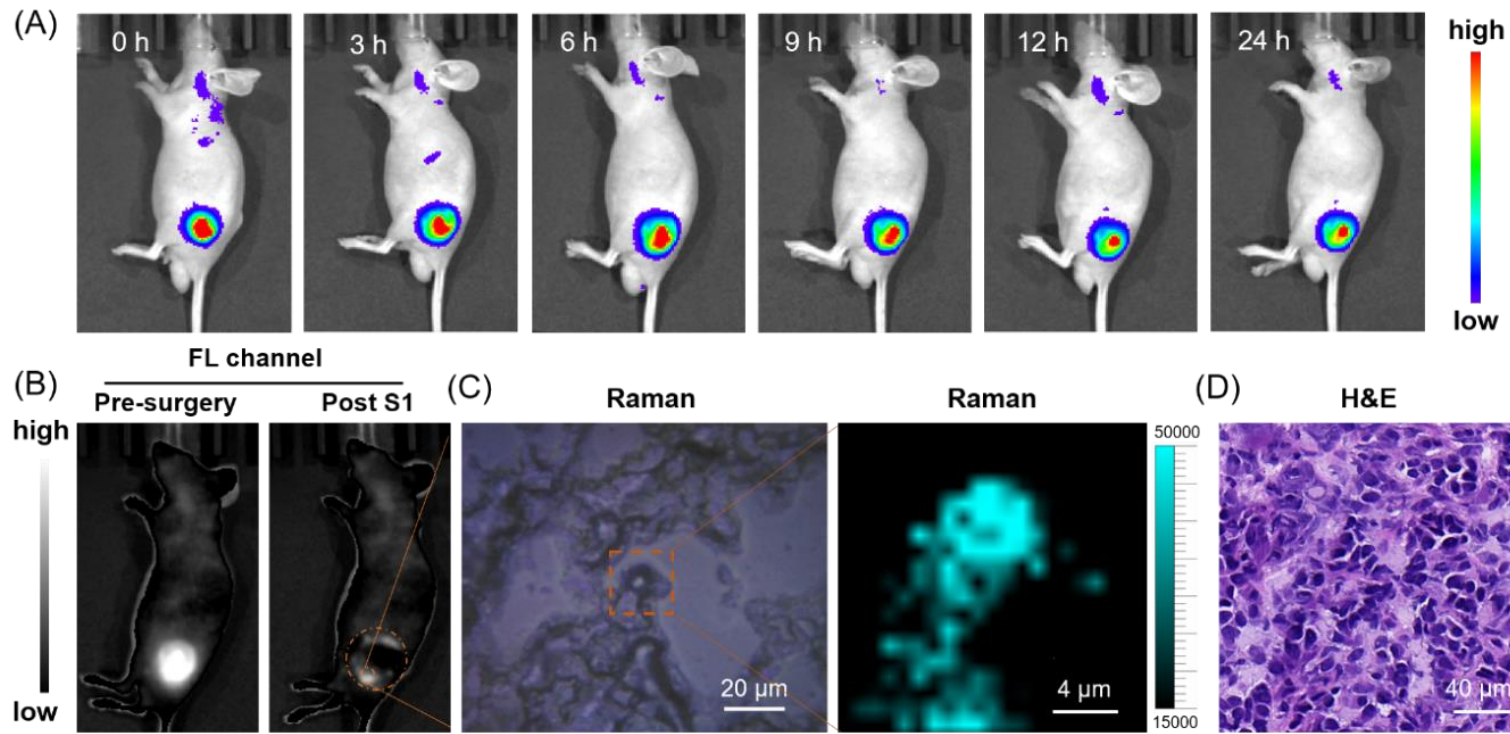

Raman
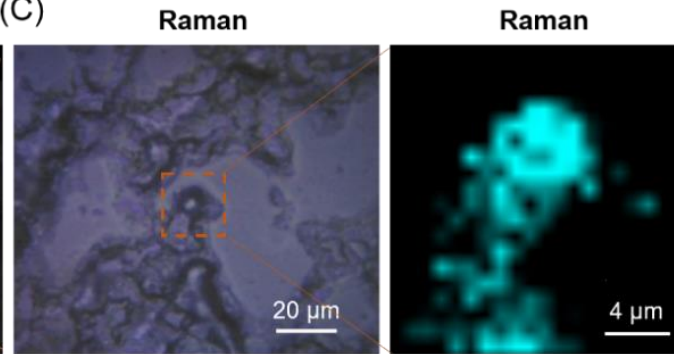

(D)

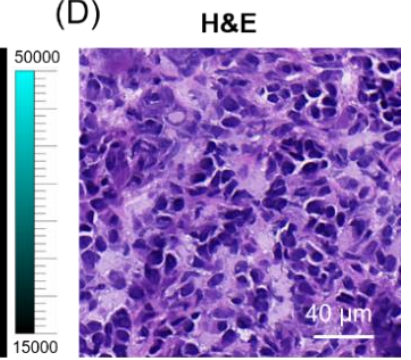

(E)

FL channel

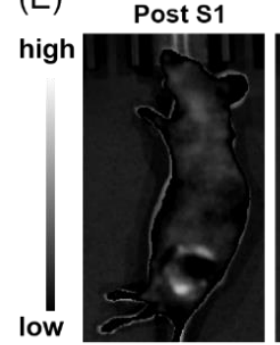

$(F)$

Raman

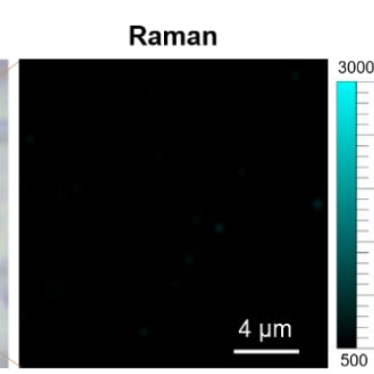

$(\mathrm{G})$

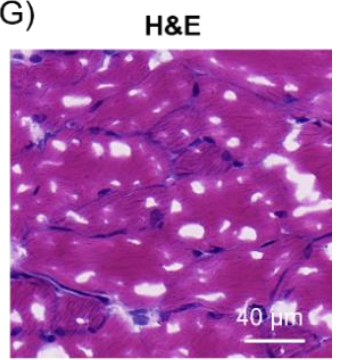

Figure 5. (A) Fluorescent images of tumor-bearing mice after intratumor injection of CP0 NPs $(50 \mu \mathrm{L}, 2 \mathrm{mg} / \mathrm{mL}$ based on CP0) at different time points as indicated. (B) Representative fluorescent images of the CP0 NPs-treated tumor-bearing mouse before and after S1 treatment. (C) Raman imaging and (D) H\&E-stained tissues of the operative incision site after S1. (E) Representative fluorescent images of the CP0 NPs-treated tumor-bearing mouse before and after S2 treatment. (F) Raman imaging and $(\mathrm{G}) \mathrm{H} \& \mathrm{E}$-stained tissues of the operative incision site after S2.

\subsection{Application in Drug-Resistant Bacteria Killing}

To develop multifunctional CPs with excellent bacteria imaging capability and efficient antibacterial activities, we then explored the application of the AIE-active CP2 with quaternary ammonium pendants in the fluorescence imaging and killing of various bacteria. The bacteria staining behaviors of $\mathbf{C P 2}$ were firstly examined using S. aureus (Gram-positive bacteria, $\mathrm{G}(+)$ ) and E. coli (Gram-negative bacteria, G(-)) as typical examples. As exhibited in Figure S12 and S13, obvious fluorescence signals 
could be observed both in $S$. aureus and in E. coli, indicating that CP2 could efficiently bind with these bacteria. Meanwhile, the bacteria tended to get together in the presence of CP2, which might result from the electrostatic and hydrophobic interactions between the cationic $\mathbf{C P 2}$ and bacterial outer membranes. ${ }^{[18-19,58]}$ Subsequently, we tested the staining performance of CP2 on bacteria that are co-incubated with normal mammalian cells (human umbilical vein endothelial cells, HUVECs). The experimental results shown in Figure 6 suggested that CP2 possessed comparatively weak binding affinity with HUVECs cells. After incubating HUVECs cells with CP2 for $30 \mathrm{~min}$, only faint fluorescence signals were detected. When HUVECs cells were mixed with $S$. aureus or E. coli and then incubated with CP2 for $30 \mathrm{~min}, \mathbf{C P 2}$ also preferred to stain bacteria over HUVECs because intensive red luminescence can be detected in S. aureus and E. coli instead of HUVECs cells. The possible reason might lie in the higher negative charge density of bacterial membrane over mammalian cells, which provided the cationic CP2 with intrinsically electrostatic driving force to bind better with bacteria. ${ }^{[51-52]}$ 


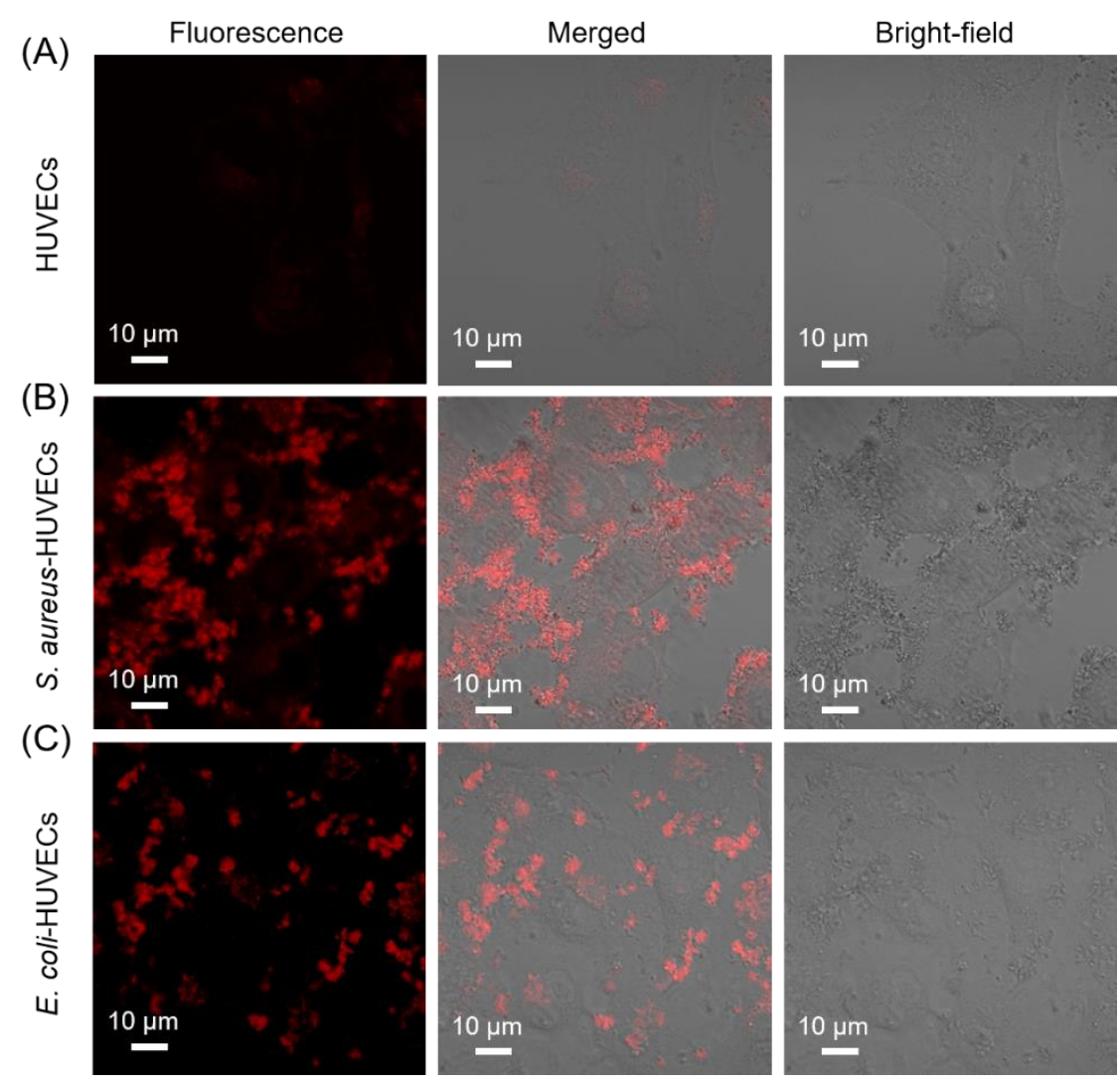

Figure 6. Fluorescent, merged and bright-field images of CP2 $(10 \mu \mathrm{g} / \mathrm{mL}, 30$ min)-treated (A) HUVECs cells, (B) HUVECs cells co-incubated with $S$. aureus for $30 \mathrm{~min}$, (C) HUVECs cells co-incubated with E. coli for $30 \mathrm{~min}$. Excitation wavelength $=405 \mathrm{~nm}$; emission wavelength $=550-700 \mathrm{~nm}$.

Encouraged by the fluorescence imaging results, we then evaluated the antibacterial performance of the AIE-active CPs. Firstly, the ROS generation ability of CP2 was investigated with 2,7-dichlorofluorescein (DCFH) as probe under white light irradiation. As a control, the ROS generation of $\mathbf{C P 0}$ was also evaluated at the same conditions. When ROS species were generated in the tested system, the DCFH will be transformed into highly fluorescent 2,7-dichlorofluorescein (DCF) to give obvious fluorescence change. As shown in Figure 7A, the fluorescence intensity at $525 \mathrm{~nm}$ (characteristic peak of DCF) monitored by a microplate reader was remarkably enhanced upon irradiating the mixture of CP2 and DCFH with white light, whereas there was no obvious increase in fluorescence intensity for the hydrophobic 
CP0 and the DCFH blank group. These results demonstrated that CP2 had potential to function as a photosensitizer to produce destructive ROS for the photodynamic killing of various bacteria. Based on these results, the bacterial survival experiments were subsequently carried out in the presence of $\mathbf{C P 2}$ with and without white light irradiation. The commonly used S. aureus $(\mathrm{G}(+)), \quad E$ coli $(\mathrm{G}(-))$ and methicillin-resistant Staphylococcu aureus (MRSA) and vancomycin-resistant Enterococcus faecium (VREF) bacteria were chosen as the representatives. As described in Figure 7B-C and Figure S14-S15, CP2 showed a certain degree of dark toxicity to all four kinds of bacteria. And the toxicity was distinctly enhanced upon 10 min irradiation of white light, especially for the S. aureus, MRSA and VREF bacteria. The survival rate of $E$. coli with the treatment of $5 \mu \mathrm{g} / \mathrm{mL} \mathrm{CP2}$ was reduced to $33 \%$ or $22 \%$ under darkness or light irradiation, respectively. More than $99 \%$ of S. aureus $(\mathrm{G}(+))$ and the drug-resistant MRSA bacteria were killed by treating them with a low concentration of $5 \mu \mathrm{g} / \mathrm{mL}$ of $\mathbf{C P 2}$ under light irradiation. The drug-resistant VREF bacteria were nearly completely killed after incubation with merely $5 \mu \mathrm{g} / \mathrm{mL}$ of $\mathbf{C P 2}$ under 10 min light irradiation. All of these results undoubtedly demonstrated the efficient photodynamic killing capability of CP2 against Gram-positive and drug-resistant bacteria. 

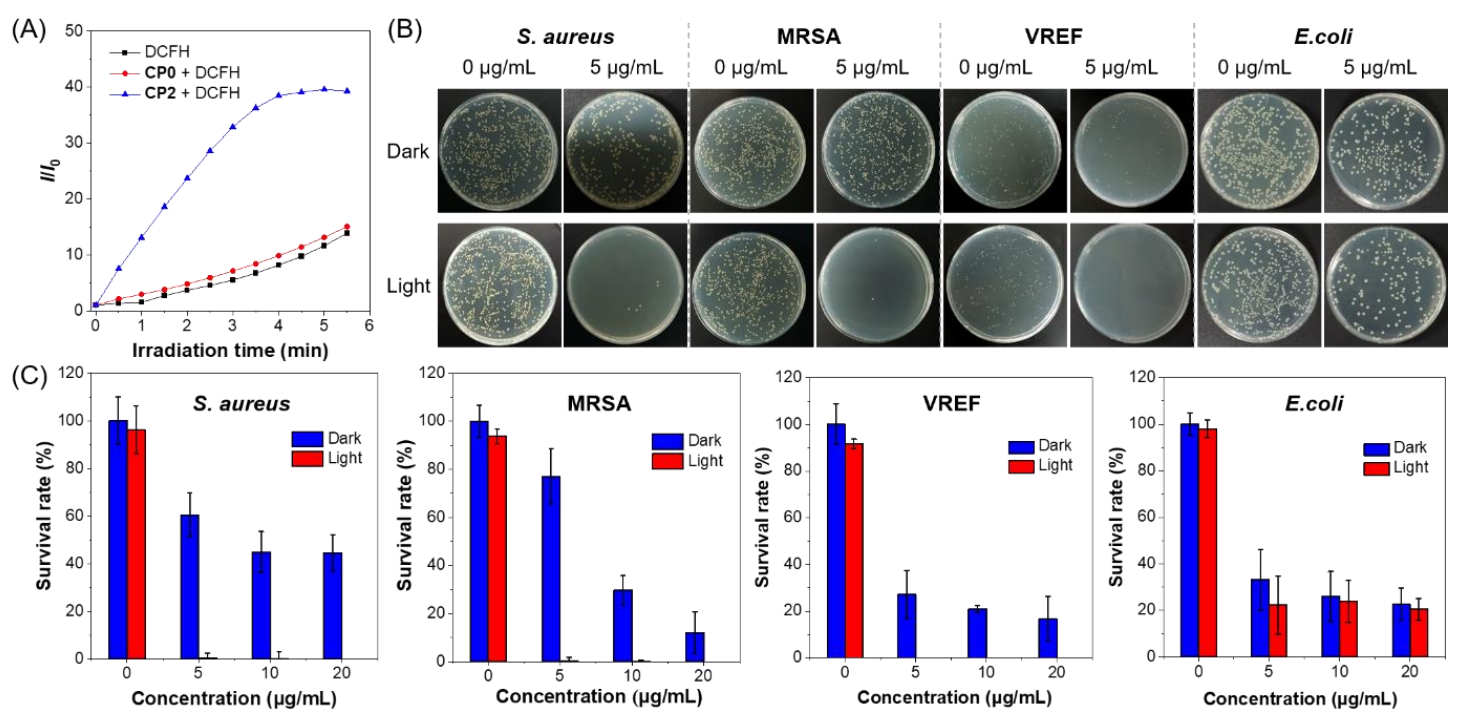

Figure 7. ROS generation and photodynamic killing of bacteria. (A) ROS generation of CP0 and CP2 $(1 \mu \mathrm{g} / \mathrm{mL})$ upon white light irradiation using DCFH as indicator. (B) Photographs of $S$. aureus, MRSA, VRE and E. coli cultured on agar plate supplemented CP2 at a concentration of $5 \mu \mathrm{g} / \mathrm{mL}$ in darkness or upon white light irradiation $\left(16 \mathrm{~mW} / \mathrm{cm}^{2}\right)$ for $10 \mathrm{~min}(\mathrm{n}=3)$. (C) The killing efficiency of $\mathbf{C P 2}$ on $S$. aureus, MRSA, VRE and E. coli.

To understand the mechanism of killing bacteria, scanning electron microscopy (SEM) and transmission electron microscopy (TEM) were employed to visualize the morphological changes of $S$. aureus under incubation with CP2. As shown in Figure 8A-B, the S. aureus with only light irradiation hold their integrity and smooth outer membranes could be clearly observed from the TEM image. After treating bacteria with $\mathbf{C P 2}$ in darkness or under light irradiation, structural damage occurred. In particular, parts of $S$. aureus collapsed under light irradiation and showed broken membrane with lots of debris on the surface of the bacteria. Membrane damage also can be observed in darkness but not as severe as under the light irradiation. These results again demonstrated that the killing of $S$. aureus mainly resulted from the PDT accompanied with a certain extent of dark toxicity. The cytotoxicity of CP2 was also evaluated by MTT assay (Figure 8C). At a concentration 
of $5 \mu \mathrm{g} / \mathrm{mL}$ CP2, no significant cytotoxicity was observed. When the concentration reached up to $20 \mu \mathrm{g} / \mathrm{mL}$, the cell viabilities of HeLa, 4T1 and HUVCEs could still remain surpass $80 \%$ either in darkness or in the present of white light irradiation (16 $\mathrm{mW} / \mathrm{cm}^{2}$ ) for $10 \mathrm{~min}$. These results further demonstrated that the cationic $\mathbf{C P 2}$ tends to bind with bacteria rather than cells. Therefore, the AIE-active CP2 is promising to be utilized for the preferred imaging of bacteria over normal cells with good cytocompatibility and can efficiently kill multidrug-resistant bacteria.
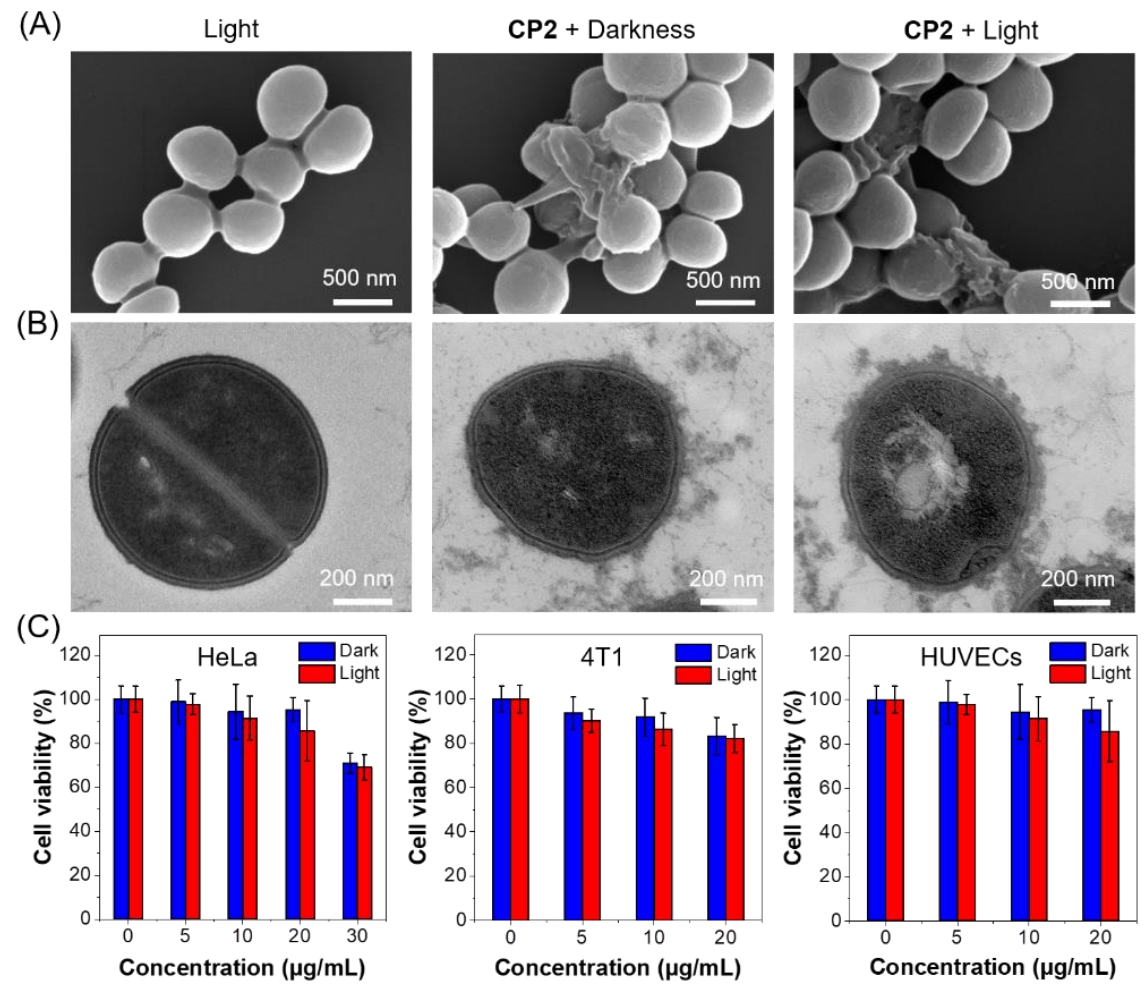

Figure 8. Visualizing morphological changes of $S$. aureus incubated with or without CP2 $(10 \mu \mathrm{g} / \mathrm{mL})$ in the darkness or upon white light irradiation $\left(16 \mathrm{~mW} / \mathrm{cm}^{2}\right)$ for 30 min by HR-SEM (A) and TEM (B), (Left) CP2+darkness, (Middle) CP2 + light, (Right) light. (C) MTT assay of HeLa cells, 4T1 cells, and HUVECs treated by different concentrations of $\mathbf{C P 2}$ in darkness or upon white light irradiation (16 $\left.\mathrm{mW} / \mathrm{cm}^{2}\right)$ for $10 \mathrm{~min}(\mathrm{n}=5)$.

\section{Conclusion}


In summary, we successfully prepared a series of AIEgen-containing PPE derivatives named CP0-CP2. These conjugated polymers all showed typical AIE properties with efficient aggregate-state fluorescence. The amphiphlic CP1 with ammonium salt groups exhibited diversiform self-assembly morphologies in water/THF mixtures and fluorescent nanofibers with helical structure can be visualized by STED nanoscopy. In addition to efficient fluorescence, the large $\pi$-conjugated structure endows these PPEs with obviously enhanced alkyne vibrations in the Raman-silent region of cells compared to alkyne-containing small molecules. CP0 exhibited a RIE value of about 17, which is much higher than the widely used Raman-active small molecules. The prepared well-dispersed CPO NPs can be applied for the real-time and fast imaging of tumors via fluorescence signals as well as the sensitive positioning of residual tumors via Raman imaging. After further modification, the obtained conjugated polyelectrolyte CP2 with quaternary ammonium pendants can preferentially image bacteria over mammalian cells with good cytocompatibility. Moreover, CP2 possessed efficient killing capability against Gram-positive and drug-resistant bacteria. The antibacterial activity of CP2 mainly resulted from the PDT accompanied with a certain extent of dark toxicity. The design strategy and the systematic study on the photophysical properties and biological activities of AIE-active PPEs in this work will benefit the further development of multifunctional conjugated polymers for multimodal imaging-guided cancer surgery and efficient photodynamic killing of multidrug-resistant bacteria. Further experiments on AIE-active polymers with near 
infrared fluorescence and more efficient Raman activities are under investigation in our lab.

\section{Supporting Information}

Supporting Information is available from the Wiley Online Library or from the author.

\section{Acknowledgements}

X.S. and R.L. contributed equally to this work. This work was supported by the National Natural Science Foundation of China (21905176, 21801169), the Natural Science Foundation for Distinguished Young Scholars of Guangdong Province (2020B1515020011), and the Science and Technology Plan of Shenzhen (JCYJ20190808142403590, JCYJ20190808153415062, RCYX20200714114525101). The authors also acknowledge the Instrumental Analysis Center of Shenzhen University and thank Dr. Tao Xue for Raman measurement at the Center for Analysis and Tests of Tianjin University.

\section{Conflict of Interest}

The authors declare no conflict of interest.

Received: ((will be filled in by the editorial staff))

Revised: ((will be filled in by the editorial staff)) Published online: ((will be filled in by the editorial staff))

\section{References}

[1] C. Zhu, L. Liu, Q. Yang, F. Lv, S. Wang, Chem. Rev. 2012, 112, 4687.

[2] T. Yamamoto, Macromol. Rapid Commun. 2002, 23, 583.

[3] W. Wu, G. C. Bazan, B. Liu, Chem 2017, 2, 760. 
[4] R. Zhan, B. Liu, Macromol. Rapid Commun. 2017, 38, 1700010.

[5] C. V. Hoven, A. Garcia, G. C. Bazan, T.-Q. Nguyen, Adv. Mater. 2008, 20, 379.

[6] M. Gross, D. C. Mgller, H.-G. Nothofer, U. Scherf, D. Neher, C. Br-uchle, K. Meerholz, Nature 2000, 405, 661.

[7] N. Stutzmann, R. H. Friend, H. Sirringhaus, Science 2003, 299, 1881.

[8] L. Lu, W. Chen, T. Xu, L. Yu, Nat. commun. 2015, 6, 7327.

[9] Z. Zhang, M. Liao, H. Lou, Y. Hu, X. Sun, H. Peng, Adv. Mater. 2018, 30, 1704261.

[10]H. Zhou, M. H. Chua, B. Z. Tang, J. Xu, Polym. Chem. 2019, 10, 3822.

[11]K. Fidanovski, D. Mawad, Adv. Healthcare Mater. 2019, 8, 1900053.

[12] Y. Jiang, J. Li, X. Zhen, C. Xie, K. Pu, Adv. Mater. 2018, 30, 1705980.

[13]J. Han, M. Bender, K. Seehafer, U. H. F. Bunz, Angew. Chem. Int. Ed. 2016, 55, 7689.

[14]A. Satrijo, T. M. Swager, J. Am. Chem. Soc. 2007, 129, 16020.

[15]R. L. Phillips, I.-B. Kim, L. M. Tolbert, U. H. F. Bunz, J. Am. Chem. Soc. 2008, $130,6952$.

[16]S. S. Khatoon, Y. Chen, H. Zhao, F. Lv, L. Liu, S. Wang, Biomater. Sci. 2020, 8, 2156.

[17]J. H. Moon, P. Manandhar, H. Torabi, M. R. Rohman, L. Mathivathanan, K. H. Lee, S. Irle, Chem. Commun. 2019, 55, 5930.

[18]Y. Wang, K. S. Schanze, E. Y. Chi, D. G. Whitten, Langmuir 2013, 29, 10635.

[19] Y. Wang, E. Y. Chi, K. S. Schanze, D. G. Whitten, Soft Matter. 2012, 8, 8547. 
[20]J. A. Osaheni, S. A. Jenekhe, J. Am. Chem. Soc. 1995, 117, 7389.

[21]J. S. Yang, T. M. Swager, J. Am. Chem. Soc. 1998, 120, 5321.

[22] J. A. Osaheni, S. A. Jenekhe, J. Am. Chem. Soc. 1995, 117, 7389.

[23]J. Mei, N. L. C. Leung, R. T. K. Kwok, J. W. Y. Lam, B. Z. Tang, Chem. Rev. 2015, 115,11718 .

[24]S. Xu, Y. Duan, B. Liu, Adv. Mater. 2020, 32, 1903530.

[25]B. R. Smith, S. S. Gambhir, Chem. Rev. 2017, 117, 90.

[26]H. Kobayashi, M. Ogawa, R. Alford, P. L. Choyke, Y. Urano, Chem. Rev. 2010, $110,2620$.

[27]H.Yamakoshi, K. Dodo, A. Palonpon, J. Ando, K. Fujita, S. Kawata, J. Am. Chem. Soc. 2012, 134, 20681.

[28]G. M. Arzumanyan, N. V. Doroshkevich, K. Z. Mamatkulov, S. N. Shashkov, E. V. Zinovev, A. V. Vlasov, E. S. Round, V. I. Gordeliy, J. Am. Chem. Soc. 2016, 138, 13457.

[29]C. Krafft, I. Schie, T. Meyer, M. Schmitt, J. Popp, Chem. Soc. Rev. 2016, 45, 1819.

[30]J. F. Li, Y. J. Zhang, S. Y. Ding, R. Panneerselvam, Z. Q. Tian, Chem. Rev. 2017, $117,5002$.

[31]D. Cialla-May, X. S. Zheng, K. Weber, J. Popp, Chem. Soc. Rev. 2017, 46, 3945.

[32]L. Wei, F. Hu, Z. Chen, Y. Shen, L. Zhang, W. Min, Acc.Chem. Res. 2016, 49, 1494.

[33]D. Fu, W. Yang, X. S. Xie, J. Am. Chem. Soc. 2017, 139, 583. 
[34]Y. Tang, Y. Zhuang, S. Zhang, Z. J. Smith, Y. Li, X. Mu, M. Li, C. He, X. Zheng, F. Pan, T. Gao, L. Zhang, ACS Cent. Sci., DOI: 10.1021/acscentsci.1c00117.

[35] S.Tian, H. Li, Z. Li, H. Tang, M. Yin, Y. Chen, S. Wang, Y. Gao, X. Yang, F.

Meng, J. W. Lauher, P. Wang, L. Luo, Nat. Commun. 2020, 11, 81.

[36]F. Hu , C. Zeng, R. Long, Y. Miao, L. Wei, Q. Xu, W. Min, Nat. Methods 2018, $15,194$.

[37]S. Li, T. Chen, Y. Wang, L. Liu, F. Lv, Z. Li, Y. Huang, K. S. Schanze, S. Wang, Angew. Chem. Int. Ed. 2017, 56, 13455.

[38]X. Li, M. Jiang, J. W. Y. Lam, B. Z. Tang, J. Y. Qu J. Am. Chem. Soc. 2017, 139, 17022.

[39]J. Qi, J. Li, R. Liu, Q. Li, H. Zhang, J. W.Y. Lam, R. T.K. Kwok, D. Liu, D. Ding, B. Z. Tang, Chem 2019, 5, 1.

[40]J. M. O. Oduor, N. Onkoba, F. Maloba, A. Nyachieo, Afr. J. Lab. Med. 2016, 5, 435.

[41]S. Tang, S. K. Biswas, W. S. Tan, A. K. Saha, B.-F. Leo, PeerJ 2019, 7, e6225.

[42]P. Makvandi, R. Jamaledinc, M. Jabbari, N. Nikfarjam, A. Borzacchiello, Dent. Mater. 2018, 34, 851.

[43]M. Magana, M. Pushpanathan, A. L Santos, L. Leanse, M. Fernandez, A. Ioannidis, M. A Giulianotti, Y. Apidianakis, S. Bradfute, A. L Ferguson, A. Cherkasov, M. N Seleem, C. Pinilla, T. Lazaridis, T. Dai, R. A Houghten, R. E W Hancock, G. P Tegos, Lancet Infect. Dis. 2020, 20, e216. 
[44]X. He, L.-H. Xiong, Z. Zhao, Z. Wang, L. Luo, J. W. Y. Lam, R. T. K. Kwok, B. Z. Tang, Theranostics 2019, 9, 3223.

[45]H. Bai, W. He, J. H. C. Chau, Z. Zheng, R. T.K. Kwok, J. W. Y. Lam, B. Z. Tang, Biomaterials 2021, 268, 120598.

[46]C. Zhu, L. Liu, Q. Yang, F. Lv, S. Wang, Chem. Rev. 2012, 112, 4687.

[47]C. A. Strassert, M. O. Dipl.-Chem, R. Q. Albuquerque, A. H. Dipl.-Biol, Y. Vida, B. Maier, L. D. Cola, Angew. Chem. Int. Ed. 2009, 48, 7928.

[48]B. Wang, M. Wang, A. Mikhailovsky, S. Wang, G. C. Bazan, Angew. Chem. Int. Ed. 2017, 56, 5031 .

[49]W. Wu, D. Mao, S. Xu, M. Panahandeh-Fard, Y. Duan, F. Hu, D. Kong, B. Liu, Adv. Funct. Mater. 2019, 29, 1901791.

[50]L. K. Ista, D. Dascier, E. Ji, A. Parthasarathy, T. S. Corbitt, K. S. Schanze, D. G. Whitten, ACS Appl. Mater. Interfaces 2011, 3, 2932.

[51]Y. Wang, T. S. Corbitt, S. D. Jett, Y. Tang, K. S. Schanze, E. Y. Chi, D. G. Whitten, Langmuir 2012, 28, 65.

[52]L. P. Ding , E. Y. Chi , K. S. Schanze , G. P. Lopez , D. G. Whitten, Langmuir $2010,26,5544$.

[53]W. Huang, M. Bender, K. Seehafer, I. Wacker, R. R. Schröder, U. H. F. Bunz, Macromol. Rapid Commun. 2019, 40, 1800774.

[54]J. Zhang, Q. Liu, W. Wu, J. Peng, H. Zhang, F. Song, B. He, X. Wang, H. H.-Y. Sung, M. Chen, B. S. Li, S. H.Liu, J. W. Y. Lam, B. Z. Tang, ACS Nano 2019, 13, 3618. 
[55]D. Dang, H. Zhang, Y. Xu, R. Xu, Z. Wang, R. T. K. Kwok, J. W. Y. Lam, L.

Zhang, L. Meng, B. Z. Tang, ACS Nano 2019, 13, 11863.

[56]E. Yashima, N. Ousaka, D. Taura, K. Shimomura, T. Ikai, K. Maeda, Chem. Rev. 2016, 116, 13752.

[57]Y. Cai, Z. Guo, J. Chen, W. Li, L. Zhong, Y. Gao, L. Jiang, L. Chi, H. Tian,

W.-H. Zhu, J. Am. Chem. Soc. 2016, 138, 2219.

[58]X. Liu, M. Li, T. Han, B. Cao, Z. Qiu, Y. Li, Q. Li, Y. Hu, Z. Liu, J. W. Y. Lam, X. Hu, B. Z. Tang, J. Am. Chem. Soc. 2019, 141, 11259 